\title{
Pénfigo sifilítico: reporte de un caso
}

\author{
Syphilitic pemphigus: a case report \\ Elvis Piñeres Mejía ${ }^{1}$, Carlos Ospina Mendieta ${ }^{1}(0)$ \\ ${ }^{1}$ Clínica Primavera. Villavicencio, Meta, Colombia.
}

\section{RESUMEN}

La sífilis congénita (SC) es el resultado de la infección producida por el Treponema pallidum al feto, en una madre con sífilis gestacional (SG). La enfermedad puede ser asintomática en el $60 \%$ de los recién nacidos (RN) afectados, siendo además difícil de diagnosticar clínica e histológicamente, porque tanto sus patrones clínicos como histológicos, pueden imitar otras enfermedades dermatológicas del RN. Siendo la SC una enfermedad prevenible, siguen presentándose casos con manifestaciones clínicas severas. Presentamos el caso de un $\mathrm{RN}$ con SC de presentación grave desde el nacimiento, con manifestaciones cutáneas que incluyeron múltiples lesiones tipo vesículas y bulas, con zonas hemorrágicas y áreas denudadas con maceración, descamación y costras, de predominio en palmas y plantas, compatibles con pénfigo sifilítico. Reconocer las diversas manifestaciones clínicas, en especial las cutáneas, es fundamental para realizar un diagnóstico oportuno de SC en RN y lactantes.

Palabras clave: Sífilis congénita, pénfigo, recién nacido.

\begin{abstract}
Congenital syphilis (CS) is the result of an fetal infection caused by Treponema pallidum, in a mother with gestational syphilis (GS). The disease can be asymptomatic in $60 \%$ of affected newborns (NBs), and is also difficult to diagnose clinically and histologically, because both its clinical and histological patterns can mimic other dermatological diseases of the NB. Since CS is a preventable disease, cases with severe clinical manifestations continue to appear. We present the case of an NB with severe CS presenting from birth, with skin manifestations that included multiple vesicle and bullalike lesions, with hemorrhagic areas and denuded areas with maceration, scaling and scabs, predominantly on the palms and soles, compatible with syphilitic pemphigus. Recognizing the various clinical manifestations, especially skin manifestations, in this disease, is essential to make a timely diagnosis of CS in newborns and infants.
\end{abstract}

Key words: Congenital syphilis, pemphigus, newborn.

\section{INTRODUCCIÓN}

La sífilis congénita (SC) representa una falla en el sistema de salud, dado que es una infección que, aunque prevenible, genera morbimortalidad infantil. Es endémica en los países de ingresos bajos y medios, con tasas más bajas en los países de ingresos altos, sin embargo, en los últimos años se ha visto un incremento progresivo de dichas tasas en países desarrollados ${ }^{(1)}$.
La SC ocurre debido a la transmisión transplacentaria de Treponema pallidum (TP) de la madre al feto, asociándose con abortos espontáneos, mortinatos, muertes neonatales, anomalías fetales y prematuridad $^{(2)}$.

Un $60 \%$ de los recién nacidos (RN) vivos afectados pueden ser asintomáticos, dificultando su diagnóstico temprano, y pueden llegar a desarrollar

Correspondencia: Elvis Eduardo Piñeres Mejía Correo: elyatassg@gmail.com

Conflicto de interés: Los autores declaran no poseer conflicto de interés.

Recibido: 11/10/2020 Aceptado:17/02/2021

DOI: https://doi.org/10.31698/ped.48012021013

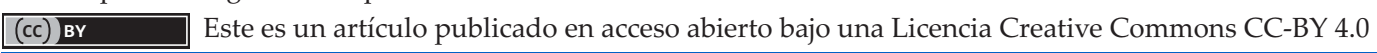


secuelas graves en etapas posteriores de su vida. El $40 \%$ restante desarrolla una enfermedad sintomática temprana, con hallazgos clínicos que pueden ser sutiles e inespecíficos, siendo difícil el diagnóstico, ya que sus patrones clínicos e histológicos pueden imitar otras enfermedades dermatológicas de los $\mathrm{RN}^{(3)}$. En escasas ocasiones, la SC puede manifestarse como una erupción vesicular-ampollar denominado pénfigo sifilítico(PS).

Presentamos el caso de un recién nacido con Sífilis congénita (SC) de presentación grave desde el nacimiento, con manifestaciones cutáneas que incluyeron múltiples lesiones tipo vesículas y bulas, con zonas hemorrágicas y áreas denudadas con maceración, descamación y costras, de predominio en palmas y plantas, compatibles con PS.

Es necesario que el personal médico asistencial se familiarice con los hallazgos clínicos de la enfermedad, y a su vez reconozca las diversas manifestaciones clínicas, en especial las cutáneas, para realizar un diagnóstico oportuno de $\mathrm{SC}$ en $\mathrm{RN}^{(4)}$.

\section{CASO CLÍNICO}

Producto de la cuarta gestación de madre de 22 años, sin controles prenatales, escolaridad $4^{\circ}$ grado primaria, vulnerabilidad económica, relación disfuncional con padre biológico, consumo de sustancias psicoactivas (Marihuana), con sífilis gestacional (SG) sin tratamiento.

Se obtiene por parto vaginal recién nacido $(\mathrm{RN})$ femenino pretérmino de 35 semanas, con medidas antropométricas peso: 2.255 gramos (p10), talla: 44 $\mathrm{cm}$ (p10), perímetro cefálico: $30 \mathrm{~cm}$ (p3), perímetro torácico: $31 \mathrm{~cm}$ (p10), perímetro abdominal $36 \mathrm{~cm}$ (p95). Con hipotonía marcada, no presenta llanto, se aseguró vía aérea con intubación orotraqueal, se acopló a ventilación mecánica asistido controlado. Adaptación conducida con APGAR de 4 y 8 al primer y quinto minuto respectivamente.

El examen físico de ingreso mostró fisuras en el área peribucal y mentón (Figura 1), abdomen distendido con hepato-esplenomegalia, (Figura 2), múltiples lesiones vesículas y bulas, con zonas hemorrágicas y áreas denudadas con maceración, algunas zonas con descamación y costras, de predominio en palmas y plantas. (Figura 3)

Exámenes de laboratorio muestra Reagina plasmática rápida (RPR) títulos 1:256 en $\mathrm{RN}, \mathrm{RPR}$ madre títulos 1:16, hemoglobina 8,6 gr/dl, plaquetas $70.000 \mathrm{~mm} 3$, bilirrubina total $9,7 \mathrm{mg} / \mathrm{dl}$, bilirrubina indirecta $8,9 \mathrm{mg} / \mathrm{dl}$, bilirrubina directa $0,8 \mathrm{mg} / \mathrm{dl}$, aspartato aminotransferasa (AST) $167 \mathrm{mg} / \mathrm{dl}$, alanino aminotransferasa (ALT) $122 \mathrm{mg} / \mathrm{dl}$, albúmina/ globulina 1,09 mg/dl, albúmina 2,4 mg/dl. La punción lumbar fue diferida por el estado de gravedad y compromiso clínico del paciente.

Radiografía de tórax Engrosamiento del intersticio peribroncovascular a nivel central por proceso inflamatorio a nivel bronquial, infiltrados de ocupación alveolar en ambos campos pulmonares (neumonía alba). (Figura 4).

El ecocardiograma evidenció signos de hipertensión pulmonar severa, con ductus arterioso permeable amplio con cortocircuito bidireccional, septum interventricular íntegro, con imagen redondeada de borde hiperecogénico (Rabdomioma) (Figura 5) en la base posterior del tabique de $7 \times 7 \mathrm{~mm}$ área de $0.9 \mathrm{~cm}^{2} \sin$ obstrucción en los tractos de entrada y salida (Figura 6).

El estudio complementario con ultrasonografía transfontanelar, radiografía de huesos largos y valoración oftalmológica, sin hallazgos patológicos. 

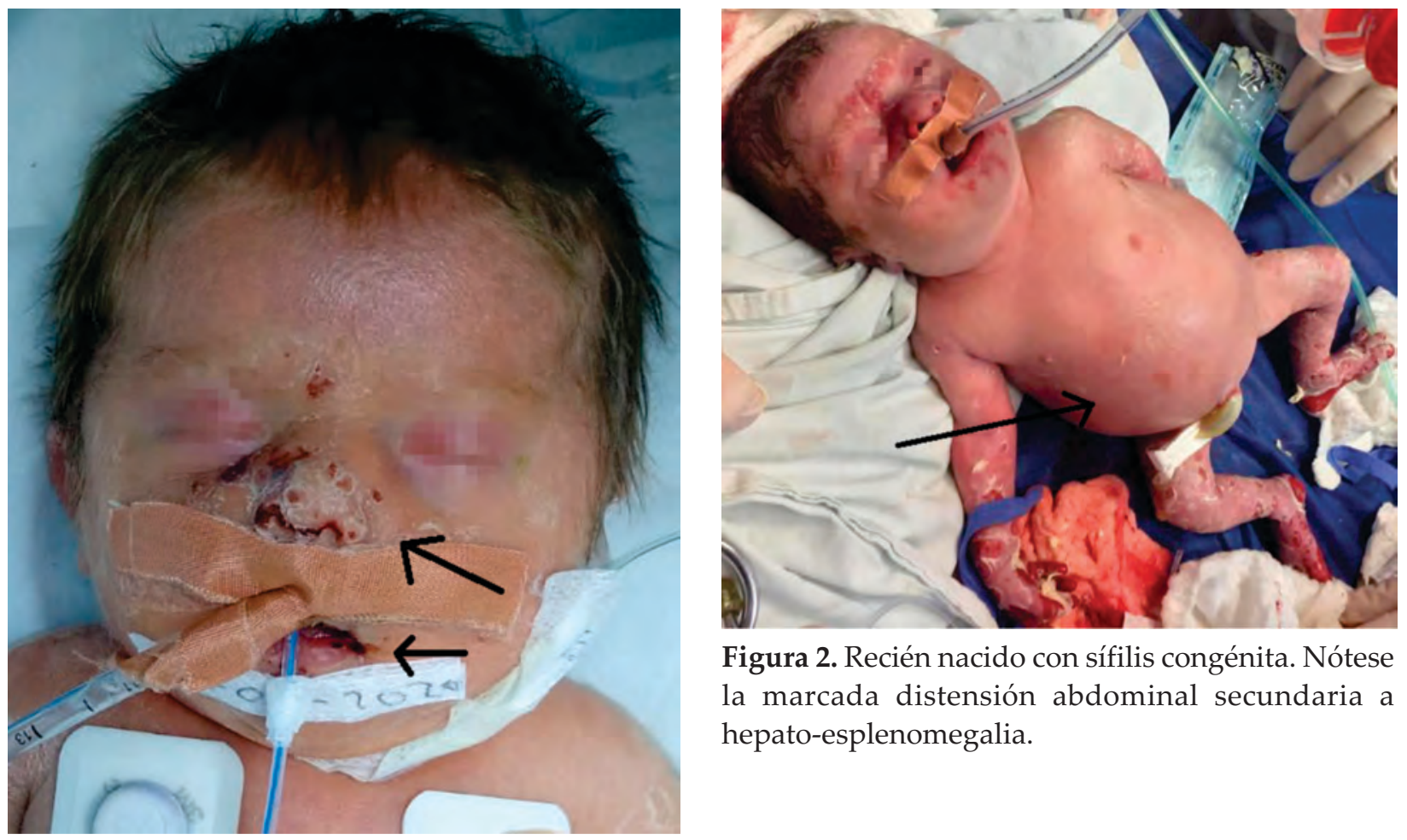

Figura 2. Recién nacido con sífilis congénita. Nótese la marcada distensión abdominal secundaria a hepato-esplenomegalia.

Figura 1. Lesiones por sífilis congénita, facies características de la enfermedad. Fisuras en área peribucal, nariz y mentón denominadas rágades.

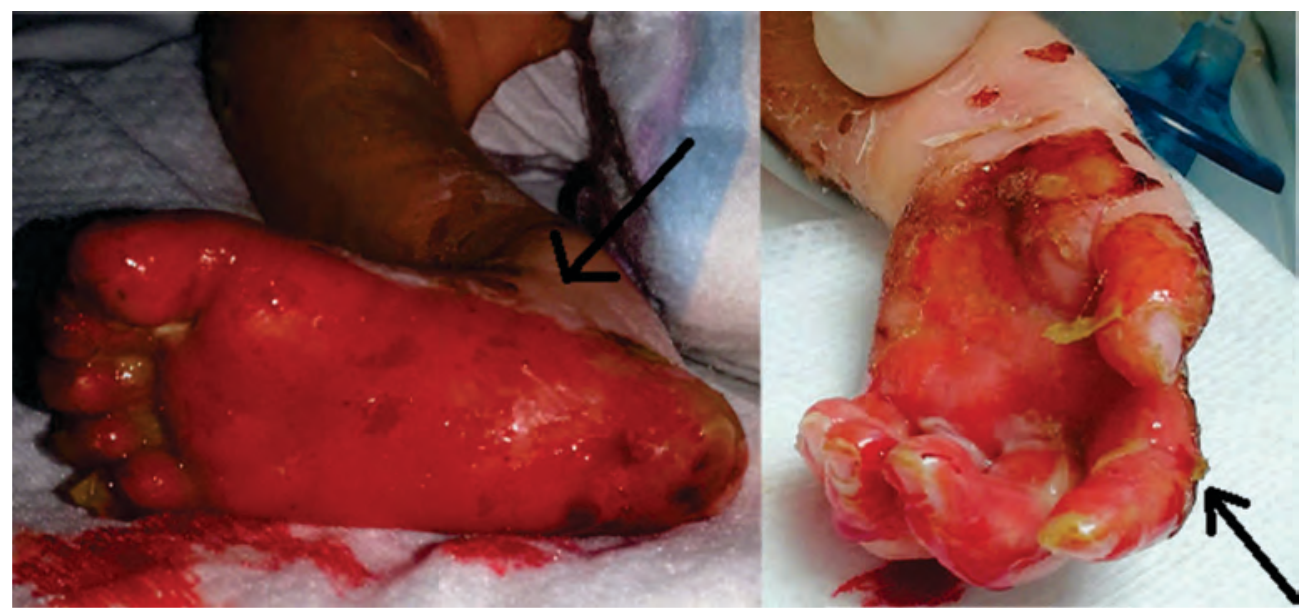

Figura 3. Lesiones en palmas y plantas denominadas Pénfigo sifilítico. Ampollas que al reventar dejan zonas hemorrágicas y áreas denudadas con maceración, zonas con descamación y costras. 


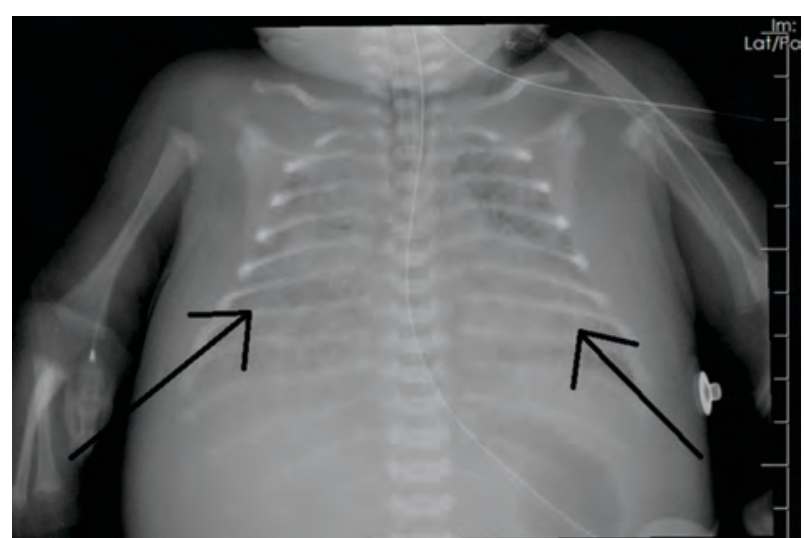

Figura 4. Radiografía de tórax. Infiltrados de ocupación alveolar en ambos campos pulmonares, denominados Neumonía alba.

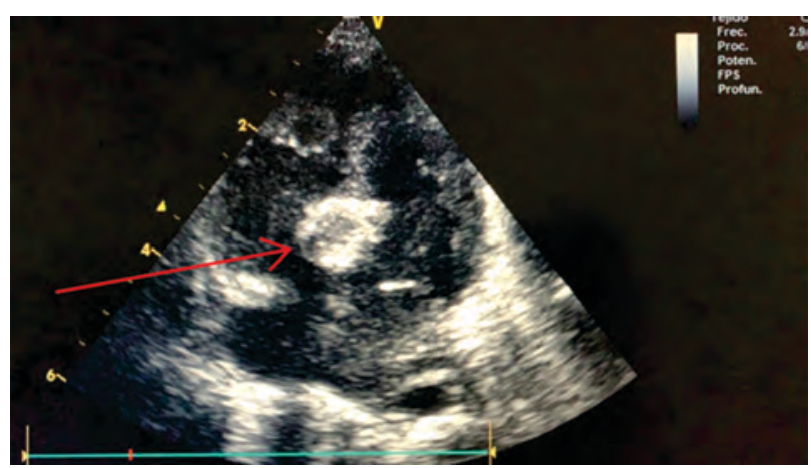

Figura 5. Ecocardiograma doppler a color. Obsérvese imagen hiperecogénica en septo interventricular (rabdomioma) sin obstrucción del flujo sanguíneo en los tractos de entrada y salida.

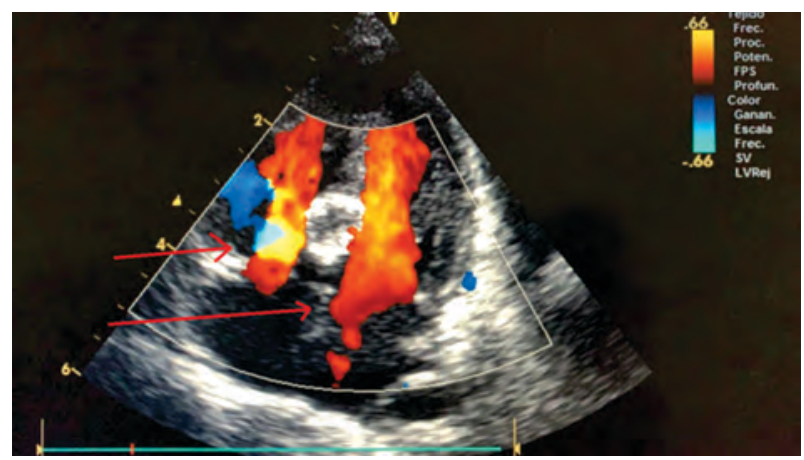

Figura 6. Ecocardiograma doppler a color. Obsérvese imagen hiperecogénica en septo interventricular (rabdomioma) sin obstrucción del flujo sanguíneo en los tractos de entrada y salida.

\section{DISCUSIÓN}

Colombia desde 2015 implementó una nueva Guía de Práctica Clínica (GPC) para la atención integral de la sífilis gestacional (SG) y sífilis congénita (SC), que incluyó el ajuste de definiciones de caso y algoritmo diagnóstico. A partir de 2016 se evidencia un incremento en la prevalencia a periodo epidemiológico (PE) VII de SG de 3,6 por 1.000 nacidos vivos (NV) en 2016 a 6,5 por $1.000 \mathrm{NV}$ en 2020, e incidencia a PE VII de SC de 0,6 por 1.000 NV en 2016 a 1,2 por $1.000 \mathrm{NV}$ en $2020^{(4)}$. Estas cifras muestran una brecha grande en la meta contemplada por la Organización Panamericana de la Salud (OPS) de reducción de SC a 0,5 casos o menos por cada $1.000 \mathrm{NV}$ para el 2020 . Un $90,6 \%$ de las madres de los casos de SC son diagnosticadas en el tercer trimestre de la gestación, sólo el $49 \%$ asiste a control prenatal (CPN), y se observa que solo el 19,9 $\%$ de madres tuvo $\mathrm{CPN}$, pero no recibieron tratamiento o fue inadecuado ${ }^{(5)}$ 苗

La madre del recién nacido de nuestro caso, presenta un bajo nivel de escolaridad y socioeconómico, nulo control prenatal, sin tratamiento durante el embarazo, abuso de sustancias psicoactivas, factores éstos, que aumentan el riesgo de presentar $\mathrm{SG}^{(6)}$.

La SC es producto de la transmisión transplacentaria de TP de la madre infectada sin tratamiento al feto, comúnmente a las 14-16 semanas de gestación o durante el parto por contacto con lesiones maternas. Se estima que la SG no tratada, tiene una probabilidad de transmisión del 70\% y aproximadamente $40 \%$ de los casos terminan en aborto, muerte fetal, muerte neonatal o parto prematuro ${ }^{(7)}$.

Cronológicamente la SC se divide según la aparición de sus manifestaciones clínicas en SC temprana, que ocurre durante los primeros 2 años de vida y SC tardía que se desarrolla posterior a los 2 años de vida. El $60 \%$ de los recién nacidos con SC pueden ser asintomáticos, dificultando su diagnóstico, el porcentaje restante desarrolla síntomas clínicos sutiles tempranos, y un porcentaje menor puede desarrollar una enfermedad sistémica aguda ${ }^{(8)}$.

Las manifestaciones clínicas de la SC temprana consisten en rinorrea, exantema macular de color 
rosado-rojo que evoluciona a color cobre de predominio en la espalda, nalgas, las palmas y plantas (similar a las manifestaciones cutáneas de la sífilis secundaria), con fina descamación, lesiones tipo pápulas alrededor de la nariz y la boca, así como en la zona del pañal, o lesiones petequiales, alteraciones óseas, alteraciones ópticas, linfadenopatías generalizadas, glomerulonefritis ${ }^{(9)}$. Puede existir una presentación grave desde el nacimiento, la forma séptica acompañada de títulos serológicos altos, marcada hepatoesplenomegalia, ictericia precoz, hepatitis luética, ascitis por hipoproteinemia, anemia severa, trombocitopenia, leucocitosis, algunas manifestaciones neurológicas como convulsiones, hemorragia intracraneal. Puede presentar además un tipo de insuficiencia respiratoria aguda por neumonitis intersticial (neumonía alba), que sí está presente en el cuadro clínico incrementa la mortalidad. A su vez, pero con menor frecuencia, las manifestaciones cutáneas son más complejas, y se presenta como erupciones tipo vesicular-ampollar con predominio en palmas y plantas, denominado pénfigo sifilítico (PS) ${ }^{(9)}$. En nuestro caso fue diagnosticado con SC título positivo de Reagina plasmática rápida (RPR) en 1:256, que era más de cuatro veces el título de la madre RPR 1:16 y presentó las manifestaciones de una SC de presentación grave desde el nacimiento y lesiones en piel compatibles con PS ${ }^{(10,11)}$.

Ante estas lesiones complejas de PS pueden establecerse como diagnósticos diferenciales: el síndrome de piel escaldada estafilocócica, epidermólisis bullosa, pénfigo ampollar vulgar, herpes gestacional, eritrodermia ictiosiforme congénita ampollar. Sin embargo, cada una de estas patologías presentan una serología, histopatología y curso clínico diferente ${ }^{(12)}$.

Desde Fournier a finales del siglo XIX, las manifestaciones clínicas cutáneas de la sífilis constituían un problema de certeza visual para los médicos. Así pues, el entrenamiento estricto a estudiantes de medicina y médicos se convirtió en un componente integral de la educación en dermatología y venereología ${ }^{(13)}$.

El personal médico asistencial debe estar habituado a las manifestaciones clínicas de la SC, comunes en otrora época, sin embargo, con la mejora en la calidad de atención, el uso de penicilina, se observan con menor frecuencia ${ }^{(14)}$.

Existe un aumento significativo en las tasas de SC en el mundo, y su control está lejos de las metas estipuladas. Reducir la transmisión materno fetal involucra no solo el autocuidado y la vigilancia, incluye también políticas efectivas que aseguren un apropiado tamizaje de mujeres durante la gestación, que las madres con SG reciban un tratamiento adecuado, tratamiento a los contactos, además de fortalecer los programas de educación a personal sanitario.

El reporte de caso permite mejor destreza en el diagnóstico clínico, manejo oportuno, así como adiestramiento a personal asistencial y en formación de una presentación de SC poco frecuente ${ }^{(15)}$. 


\section{REFERENCIAS}

1. Cooper JM, Sánchez PJ. Congenital syphilis. Semin Perinatol. 2018;42(3):176-184. doi: https://doi.org/10.1053/ j.semperi.2018.02.005

2. Mathews J, Naseem JA, Neupane N, Parameswaran N, Chandrashekar L. An unusual presentation of early congenital syphilis with annular configuration of blisters resembling "string-of-pearls". Pediatr Dermatol. 2019;36(5):735-736. doi: https://doi.org/10.1111/pde.13880

3. Spaccarelli N, Wan J, Yan A, Bhatti T, Rubin AI. Congenital syphilis as a clinical and histopathologic mimic of neonatal lupus. J Cutan Pathol. 2018;45(10):791793. doi: https://doi.org/10.1111/cup.13310

4. Raposo I, Machado S, Sampaio R, Selores M. Infantile bullous pemphigoid with "string of pearls sign". Dermatol Online J [Internet]. 2017 [cited 2020 Nov 23];23(7):13030 /qt3fw $4 \mathrm{~d} 2 \mathrm{t} 2$.

5. Organización Mundial de la Salud. Plan de acción para prevención y control de infección por el VIH y las infecciones de transmisión sexual 2016-2021 [Internet]. OMS; 2016 [cited 2020 Nov 23]. Available from: https://iris.paho.org/bitstream/handle/10665.2/34079/DC 552017-spa.pdf?sequence $=1$ \&isAllowed $=y$

6. Rowe CR, Newberry DM, Jnah AJ. Congenital Syphilis: A Discussion of Epidemiology, Diagnosis, Management, and Nurses' Role in Early Identification and Treatment. Adv Neonatal Care. 2018;18(6):438-445. doi: https://doi.org/10.1097/ANC.0000000000000534

7. Korenromp EL, Rowley J, Alonso M, Mello MB, Saman Wijesooriya N, Guy Mahiané S, et al. Global burden of maternal and congenital syphilis and associated adverse birth outcomes-Estimates for 2016 and progress since 2012. PLoS ONE, 2019; 14: e0211720-e0211720 doi: https://doi.org/10.1371/journal.pone.0211720

8. Lafetá KRG, Martelli Júnior H, Silveira MF, Paranaíba LMR. Sífilis materna e congênita, subnotificação e difícil controle. Rev Bras Epidemiol. 2016;19(1):63-74. doi: https://doi.org/10.1590/1980-5497201600010006
9. Wang EA, Chambers CJ, Silverstein M. A rare presentation of congenital syphilis: Pemphigus syphiliticus in a newborn infant with extensive desquamation of the extremities. Pediatr Dermatol. 2018;35(2):e110-3. doi: http://doi.wiley.com/10.1111/pde.13380

10. Patel NU, Oussedik E, Landis ET, Strowd LC. Early Congenital Syphilis: Recognising Symptoms of an Increasingly Prevalent Disease. J Cutan Med Surg. 2018; 22(1):97-99.doi: https://doi.org/10.1177/1203475417728360

11. Wang C, He S, Yang H, Liu Y, Zhao Y, Pang L. Unique manifestations and risk factors of Jarisch-Herxheimer reaction during treatment of child congenital syphilis. Sex Transm Infect. 2018;94(8):562-4. doi: https://doi.org/10. 1136/sextrans-2016-053083

12. Leung AKC, Leong KF, Lam JM. A Case of Congenital Syphilis Presenting with Unusual Skin Eruptions. Case Rep Pediatr. 2018;2018:1-4. doi: https://doi.org/10.1 $155 / 2018 / 1761454$

13. Pierce K. Photograph as Skin, Skin as Wax: Indexicality and the Visualisation of Syphilis in Fin-de-Siècle France The William Bynum Prize Essay. Med Hist. 2020; 64(1):116-141. doi: https://doi.org/10.1017/mdh.2019.79

14. Tang H, Zhao P, Chen J, Liu L. Congenital pemphigus syphiliticus. Int J Infect Dis. 2016; 49:149-50. doi: https://doi.org/10.1016/j.ijid.2016.05.031

15. Garcés JP, Rubiano LC, Orobio Y, Castaño M, Benavides E, Cruz A. La educación del personal de salud: clave para la eliminación de la sífilis congénita en Colombia. Biomédica. 2017; 37(3):416-24. doi: https://doi.org/10.7705/biomedica.v37i3.3397 